SECOND REVISED VERSION

\title{
Choosing small sets of policy-relevant scenarios by combining vulnerability and diversity approaches ${ }^{1}$
}

\begin{abstract}
Computer simulation models can generate large numbers of scenarios, far more than can be effectively utilized in most decision support applications. How can one best select a small number of scenarios to consider? One approach calls for choosing scenarios that illuminate vulnerabilities of proposed policies. Another calls for choosing scenarios that span a diverse range of futures. This paper joins these two approaches for the first time, proposing an optimization-based method for choosing a small number of relevant scenarios that combine both vulnerability and diversity. The paper applies the method to a real case involving climate resilient infrastructure for three African river basins (Volta, Orange and Zambezi). Introducing selection criteria in a stepwise manner helps examine how different criteria influence the choice of scenarios. The results suggest that combining vulnerability- and diversity-based criteria can provide a systematic and transparent method for scenario selection.
\end{abstract}

\section{Keywords}

Scenario diversity analysis, vulnerability based scenario analysis, climate change, scenario discovery, robust decision making

\section{Highlights}

- Describes an optimization-based method for choosing a small number of scenarios.

- A combination of criteria related to vulnerability and diversity is used.

- The method is applied to a real case involving climate resilient infrastructure.

\footnotetext{
${ }^{1}$ Part of a TI on “Innovative Techniques for Quantitative Scenarios in Energy and Environmental Research".
} 


\section{SECOND REVISED VERSION}

\section{Introduction}

Energy and environmental change planning and assessments at the local, regional, and global scales often use scenarios (Rounsevell \& Metzger 2010). For example, the Special Report on Emissions Scenarios (SRES) provides a well-known and much used scenario set commissioned by the Intergovernmental Panel on Climate Change (IPCC) (Nakicenovic et al. 2000). The SRES scenarios are explorative storylines describing future possible societies and associated greenhouse gas emissions pathways. New scenarios called Representative Concentration Pathways (RCPs; van Vuuren et al. 2011) and Shared Socio-economic Pathways (SSPs; O'Neill et al. 2015) have now replaced the SRES scenarios. The SRES, RCP, and SSP scenarios were all chosen through processes that relied largely on expert judgment. In this paper we propose a new computer-aided approach for choosing scenarios for environmental research and policy. This approach combines two perspectives on scenario development: scenarios for illuminating vulnerabilities of proposed policies and scenarios that span a diverse range of futures.

The scenario literature offers several useful taxonomies useful for organizing and comparing the many approaches for developing and using scenarios (see e.g. Bradfield et al. 2005, Börjeson et al. 2006 and Bishop et al. 2007). Here it proves useful to highlight two approaches, Intuitive Logics and Morphological Analysis, which help contextualize important features of the vulnerability and diversity approaches of interest in this paper.

Intuitive Logics (Kahn and Wiener 1967, Wilkinson and Kupers 2014) provides the most widely used technique for developing scenarios in environmental research. While there exist many variants of this school of thought, most include three key elements: a decision the scenarios are meant to inform, identification of a small number of key driving forces, and storylines (Lempert 2013). In this primarily qualitative approach, practitioners compile a list of driving forces in the external environment that may affect the decisions. Using expert judgement, practitioners then select a small number (often two) of the most important driving forces using a pair of criteria: importance to the decision and the degree of uncertainty. Each driving force generally takes on one of a small number of values (often two, e.g. 'high' and 'low') - that are then used to define a small set of scenarios (often four). Intuitive Logics seeks a small set of scenarios so users can more easily consider and compare them. The driving forces are also used to help craft a narrative 


\section{SECOND REVISED VERSION}

for each scenario that helps enhance their communicative power. Intuitive Logics generates scenarios consistent with a typical definition, such as that offered by the Millennium Ecosystem Assessment, which describes a scenario as a "plausible and often simplified description of how the future may develop, based on a coherent and internally consistent set of assumptions about key driving forces" (Millennium Ecosystem Assessment 2005, 214).

Morphological Analysis exemplifies a different approach for generating a different type of scenario (Zwicky 1969, von Reibnitz 1988, Gausemeier et al. 1998, Ritchey 2006, Scholz and Tietje 2002, Tietje 2005). The aim of Morphological Analysis is to address multi-dimensional, non-quantifiable problems by explicitly exploring all the many combinations of many 'uncertainties' (cf. 'driving forces' above ) that can each take an arbitrary number of 'states' (cf. 'values' above). The analysis then eliminates the inconsistent state combinations using a cross consistency analysis and retains the rest. Morphological Analysis thus generates many 'scenarios', each consisting of one of the plausible combinations of the many uncertainties.

Computer aided methodologies provide the foundation for Morphological Analysis (Gordon and Hayward 1968) and have also been employed to help choose scenarios at least since the 1970s, for instance within the French school La Prospective (Godet 2000). Such computer-aided methodologies have been widely used in technological forecasting, but have to a large degree been left unnoticed by the environmental research community, which tends to employ Intuitive Logics-based methods. One notable example where computer aided techniques have been used in environmental research is the 'story and simulation' (SAS) approach, where narrative storylines are used as inputs to quantitative modeling e.g. of climate change impacts (Alcamo 2001, 2008). But, in SAS computer techniques enter only after expert judgment has identified the scenarios and their storylines. Only a few examples exist in which computer aided techniques have been utilized in environmental research to identify narrative scenarios (see e.g. Schweizer and Kriegler 2012, Schweizer and O’Neill 2014, Cervignig et. al. 2015, Groves et. al. 2013).

This paper focuses on computer-aided methods for identifying exploratory scenarios for energy and environmental research and decision-making. In the terminology of Börjeson et al. (Börjeson et al. 2006), exploratory scenarios ask the question "what might happen?" and are typically used for cases, often at longer time-frames, for which the future is less predictable. Since in general an 


\section{SECOND REVISED VERSION}

unlimited number of events might happen, a key challenge with using exploratory scenarios is choosing a set of scenarios sufficiently small to be manageable yet sufficiently well-chosen to avoid missing plausible future events that would prove relevant to the purposes of the analysis. For instance, energy and environmental decision support applications often require only a small number of scenarios because users can pay close attention to only a handful. In addition, subsequent analysis conducted with the scenarios, for instance evaluating the performance of policies, may create computational constraints that also limit the number of scenarios that can be considered. However, a limited number of scenarios can also exclude futures decision makers might find important to consider.

This paper addresses this challenge by combining two computer-aided approaches for selecting energy and environmental scenarios. The first approach selects scenarios that illuminate vulnerabilities of proposed policies. This vulnerability-based approach uses simulation models to explore the performance of policies over a wide range of futures and then chooses a small number of scenarios that best distinguish those futures in which a policy meets and misses its goals (Groves \& Lempert 2007, Bryant \& Lempert 2010). The second approach selects scenarios that are diverse by identifying a small set of scenarios that best span a wide a range of futures (Carlsen et al. 2016).

Both approaches begin with a large number of plausible futures, often generated by simulation models, similar to scenarios in the Morphological Analysis sense of the term. The diversity-based approach then uses optimization algorithms to choose a small set of such scenarios that best represents the range of plausible futures. In contrast, the vulnerability-based approach uses statistical algorithms to identify clusters of futures meant to be analogous to scenarios in the Intuitive Logics sense of the term. As described in Section 2.3, combining these two previously distinct yet complementary approaches provides useful and important synergies. In joining these two approaches, this paper offers an innovative way of choosing a small and manageable number of relevant scenarios based on both vulnerability and diversity. This paper demonstrates the approach in a test case, and suggests how it could be widely useful in many applications.

The next section defines the characteristics of the scenario vulnerability and diversity approaches and discusses the relationship between them. Section 3 applies the proposed approach to a real 


\section{SECOND REVISED VERSION}

case involving climate resilient infrastructure for three African river basins (Volta, Orange and Zambezi). Section 4 concludes with pros and cons of the proposed combined approach, possible applications as well as possible extension including consistency-based approaches for systematic scenario generation, and an outlook for future research.

\section{Methods}

Why is it beneficial to combine a vulnerability approach and a diversity approach when identifying small sets of policy relevant scenarios? In order to answer this question, we first need to describe the two methodological building blocks and highlight some key similarities and differences.

\subsection{Choosing Scenarios That Illuminate Vulnerabilities}

Scenarios can be chosen to highlight the choice among alternative policy options. For instance, if an organization has an existing plan, scenarios might be chosen to stress-test that plan to identify futures in which the plan may not meet its goals. If an organization is choosing among alternative plans, scenarios might be selected to help choose the plan that seems most robust (van der Heijden 1996). Many scenario planning exercises ultimately have this type of goal. For instance, Intuitive Logics pioneer Peter Schwartz's guide to developing scenarios (1996) begins with steps that identify key decisions and then lists key factors which might influence the decisions' success or failure. While many Intuitive Logics exercises are qualitative, quantitative methods such as the vulnerability-based methods discussed in this paper can help identify scenarios that seem particularly relevant to choices decision-makers face. As one advantage over qualitative approaches, quantitative methods can help make the choice of scenarios more reproducible and traceable, more connected to decision, less ambiguous, less seemingly arbitrary and biased (Parker et al. 2015).

In particular, the concept of choosing scenarios that illuminate vulnerabilities of proposed policies (Groves and Lempert 2007, Bryant and Lempert 2010, Lempert 2013) provides one important example of such a quantitative approach to scenario selection. This Robust Decision Making (RDM) (Lempert etal. 2003; Lempert and Collins 2007) process begins with a computer 


\section{SECOND REVISED VERSION}

simulation model that projects the performance of one or more policy options, using one or more outputs of interest, contingent on various uncertainties in the model structure and input parameters. The simulation is run thousands to millions of times over an experimental design that samples different combinations of values for the uncertain model input parameters. This process generates a large database of simulation results, where each case tracks the performance of a policy option along one potential path into the future. Some criterion applied to the model outputs distinguishes those futures where the policy meets its goal from those futures where it does not. Statistical scenario discovery algorithms (Bryant and Lempert 2010, Gerst et al. 2013, Kwakkel et al. 2013) applied to the resulting multi-dimensional database then identify combinations of constraints on a small number of input parameters that best distinguish those futures where the policy meets and misses its goals.

As described in Lempert (2013), the resulting clusters of futures merit the name "scenario" in the Intuitive Logics sense because properly visualized and described, they can help focus decision makers' attention on the uncertain future conditions most important to the challenges they face and help them to identify and evaluate potential improvements and alternatives to the proposed policy. The input parameters describing the clusters become the scenarios' key drivers, similar to how that term is used in Intuitive Logics approaches. For instance, the approach was used to support the recent Colorado River Basin Supply and Demand Study, in which the U.S. Bureau of Reclamation, the seven U.S. states, and other members of the Colorado Compact reached consensus on the potential mismatch between water supply and demand in the basin over the next fifty years and evaluated opportunities for resolving these imbalances (Bureau of Reclamation 2012). Water can prove a contentious issue in the American West and the parties to this study brought a wide range of differing interests and expectations about the future, including future water demand, the effect of climate change on water supply, and how water might be allocated in future extreme conditions. By using scenario discovery methods to illuminate the vulnerabilities of the Basin's current management plan, the study was able to generate consensus on the key drivers for future imbalances and, at least as importantly, consensus on the factors that seem less important (Groves et al. 2013). 


\section{SECOND REVISED VERSION}

\subsection{Choosing Scenario Sets That Are Diverse}

Another approach focuses on finding scenarios, in the Morphological Analysis sense of the term, that span a broad range of possible futures. As shown in Figure 1, a scenario set can fail to be diverse in two ways (Carlsen et al. 2016). They can be: 1) too conservative, with all scenarios close together, often around some type of 'business-as-usual' future, or 2) unbalanced, in the sense that the set contains extreme hence challenging scenarios in some 'directions' and unchallenging ones in others.

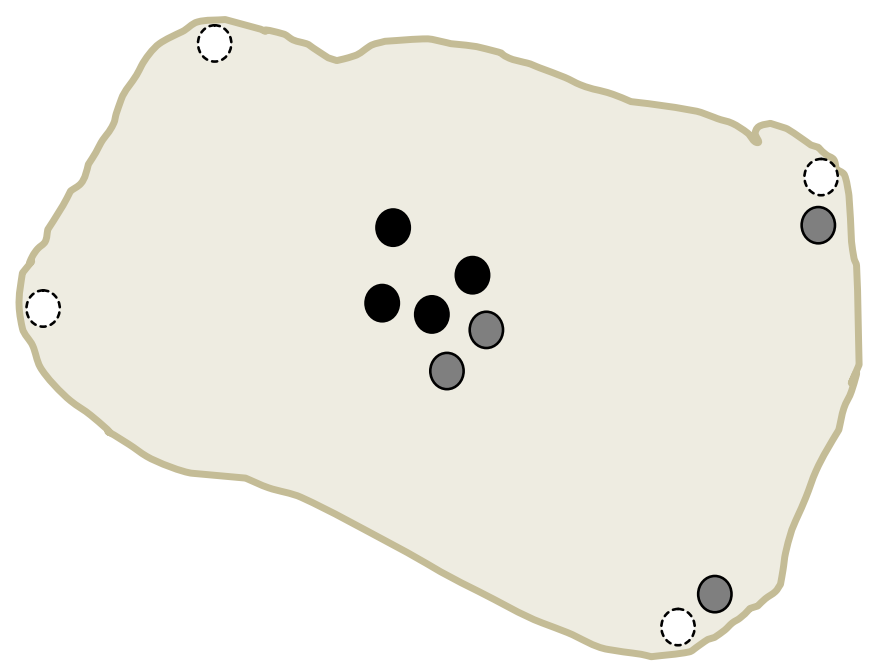

Figure 1: Schematic illustration of three archetypes of scenario sets: The black dots represent a conservative scenario set, the grey dots represent an unbalanced set and the white dashed dots represent a diverse set of scenarios (adapted from Carlsen et al. 2016).

But how can a small scenario set be selected so that it spans as wide a range (in Figure 1, the white dashed dots) of future developments as possible? The scenario diversity approach defines a measure of distance between pairs of scenarios and uses a numerical optimization algorithm to find a set of scenarios that maximizes the sum of all distances between the scenarios. Scenarios are here expressed by means of variables. Each variable is typically defined to be discrete, assuming a finite number of mutually exclusive states $s_{i}$. With $n$ variables a scenario is an $n$-tuple of states. 


\section{SECOND REVISED VERSION}

The generic methodology for scenario diversity consists of three steps normally executed in several iterations. Each iteration may, for example, add or delete new variables and/or states, as the understanding of the problem increases.

The first step rules out inconsistent state combinations. The space of potential scenarios is the whole cross-product set of state combinations (before any inconsistent combinations have been identified). In this step of the process, any standard way of reducing the number of possible scenarios can be utilized (cf. Morphological Analysis in the Introduction). This self-consistency step is discussed in more detail in Section 4.

The second step introduces a metric for the distance between any two scenarios. The intuition behind the metric is that the distance between two scenarios is large when the distances between the states for each variable are large. This intuition is captured by the weighed Manhattan distance $d$ between scenario $x_{i}$ and $x_{j}$ with $K$ variables:

$$
d\left(x_{i}, x_{j}\right)=\sum_{k=1}^{K} w_{k}\left|s_{k}\left(x_{i}\right)-s_{k}\left(x_{j}\right)\right|
$$

where $w_{k}$ describes weights imposed to reflect the importance of variable $k$ and $s_{k}\left(x_{i}\right)$ is the state of variable $k$ for scenario $x_{i}$ The possibility of using different weights for different variables gives the opportunity to reflect the relative importance of the variables, based on e.g. stakeholder prioritization in a participatory scenario building process (Kok et al. 2006, Carlsen et al. 2013). The weights best reflect the distance among scenarios if all variables are given in a common scale. In the example in this paper, we accomplish this by placing all the variables on a common, -1 to 1 , scale ranging from the smallest to largest values.

The third step generates maximally diverse scenario sets. The objective function for this optimization uses two measures. The first is the minimal distance between scenarios:

$$
D_{\min }\left(x_{1}, \ldots, x_{n}\right)=\min _{i, j} d\left(x_{i}, x_{j}\right)
$$

and the second the mean distance between the scenario sets: 


\section{SECOND REVISED VERSION}

$$
D_{\text {mean }}\left(x_{1}, \ldots, x_{n}\right)=[n(n-1) / 2]^{-1} \sum_{i=1}^{n} \sum_{j=i+1}^{n} d\left(x_{i}, x_{j}\right) .
$$

The first measure, $D_{\min }$, is relevant in this optimization because scenario sets with two or more respective scenarios too close to each other should ideally be excluded from the selected set. In most applications it will be of interest to identify scenario sets with a high value of the mean distance between all respective pairs of scenarios as well as securing that the minimum distance is not too small. The optimization typically does not yield a unique result. It generally yields many equally diverse sets. This feature of indeterminacy could be used for some flexibility when combining this methodology with other approaches, as will be demonstrated below in Section 3 .

In scenario diversity analysis (Carlsen et al. 2016 ) there is an important distinction between variables that are nominal and those that are ordinal. The states of an ordinal variable, in contrast to a nominal one, can be ordered in a meaningful way, e.g. the states of the variable 'Energy use' could quite naturally be ordered (e.g. as High $>$ Medium $>$ Low). The difference between an all nominal case and a case with some ordinal variables can prove important. For example, the former specification can result in very large numbers of scenario sets being lumped together by the distance metric into a few huge groups. To see this, consider first an ordinal variable with three states using the scale above (i.e. -1,0,1). For this variable there are three different distances between the states: 0,1 and 2. A nominal variable with three states only have two distances: 0 if the states coincide and $c$ if the states are different (for example $c=1$ or $c=2$, dependent on the chosen scale). Working with ordinal variables implies much more distinct optima compared to an all-nominal problem specification. For problems where some variables can be meaningfully ordered we therefore recommend exploring this additional structure in order to identify distinct diverse scenario sets (note that for variables that have only two states there is no difference between an ordinal and nominal specification). 


\section{SECOND REVISED VERSION}

\subsection{Comparing Vulnerability and Diversity Approaches for Scenario Selection}

Vulnerability-based and diversity-based approaches present important differences in focus.

Diversity-based approaches typically consider policy options as external to the scenarios that are analyzed for diversity, i.e. the diversity measure does not consider how diverse the outcome of different policies are over a range of scenarios. In contrast, vulnerability-approaches make the policy options under consideration central to the scenario selection. Hence scenario diversity analysis generally represents a policy independent approach for scenario selection while the vulnerability approach represents a policy dependent approach. Not all scenario discovery applications seek to identify the vulnerabilities of proposed policies nor need be policy dependent. For instance such methods have been used to identify key drivers of the IPCC Shared Socio-economic Pathways (SSPs) (Rozenberg et al. 2013), alternative levels of emissions/energy futures (Gerst et al. 2013), or different patterns of temporal dynamics (Kwakkel et al. 2013). Thus the combination of diversity and scenario discovery-based approaches likely has broader application than that described in this paper. In addition and as noted above, the vulnerability approach often generates scenarios in the Intuitive Logics sense of the term while diversity approach generates scenarios in the Morphological Analysis sense. But the two approaches also have important similarities. The scenarios that illuminate a policy's vulnerabilities are often diverse. Conversely, seeking diverse scenarios that span the extreme outcomes often proves policy relevant, since the performance of policies in 'extreme' cases is often of interest.

The approaches can usefully complement each other. For example, a vulnerability analysis generates clusters of futures that represent a scenario. However, it may prove useful to find representative cases within each cluster if, for instance, computationally expensive analysis of the scenarios is required. Scenario diversity analysis could be used to select such representative cases (the analysis can generate several different criteria, see Section 3 and Table 2 below). Conversely, scenario diversity analyses may result in many different scenarios that are equally diverse. The problem then arises how to choose between different, equally diverse, scenario sets. Here a vulnerability-based analysis could provide additional criteria by ranking different scenarios based on how policy-relevant they are, e.g. by illuminating specific vulnerabilities of alternative plans and strategies. 


\section{SECOND REVISED VERSION}

There thus seems to be significant potential in exploring how vulnerability-based and diversitybased scenario analyses can be combined in a more methodical way. In the next section we experiment with different ways of combining these approaches based on scenario sets drawn from a real application.

\section{Case Study: Enhancing the Climate Resilience of Africa's Infrastructure}

In order to demonstrate the combination of a vulnerability-based and a diversity-based approach for selecting a small number of scenarios, we consider a case study involving climate resilient infrastructure for three African river basins. This section starts with a description of the context of the case study. The combined vulnerability-diversity approach is then introduced by adding additional criteria in a stepwise manner. This sequence reveals some of the prevailing complexities in combining different scenario development and selection methodologies.

\subsection{Background to the Case Study}

Many African nations and international donors have plans for significant, multi-billion dollar investments in energy and water infrastructure over the next few decades. While necessary to continue improving the continent's quality of life and economic development, current investment plans have yet to account for the potential impacts of climate change, which may significantly affect hydropower availability, demand for irrigation and energy, and many other factors relevant to these investments' success. A recent World Bank report addressing this challenge, Enhancing the Climate Resilience of Africa's Infrastructure (ECRAI) (Cervigni et al. 2015), provides a good test bed for exploring the combination of scenario diversity and vulnerability approaches.

The ECRAI report assesses the impacts of climate change on hydropower and irrigation investment plans in seven of Africa's main river basins - the Congo, Niger, Nile, Orange, Senegal, Volta, and Zambezi - and finds that the impacts can be large, up to 60 percent of hydropower revenue in some cases. The report then identifies adaptation actions that can significantly reduce these vulnerabilities. In assessing the impacts, the report considers 121 alternative climate futures, assembled from those used in both the Fourth and Fifth IPCC 


\section{SECOND REVISED VERSION}

Assessment Reports (that is, the CMIP3 and CMIP5 ensembles) using two different downscaling techniques (Boehlert et al. 2015). However, identifying potential adaptations required running optimization algorithms on complicated river basin simulation models, which limited the analysis to considering no more than six climate futures per river basin. Because the report examined adaptations that shared risk among basins (for instance, electric transmission lines that would move hydropower generated in wet regions to dry ones) it needed to choose six representative climate futures for the entire continent.

The ECRAI report used a relatively crude approach for selecting these six representative climate futures. In brief, analysts calculated the CMI (climate moisture index) for each climate future in each basin. The analysis then ran a search looking for sets of six futures that had at least one low, mid, and high CMI value for each basin. The report defined a low value as a CMI among the six lowest of futures (5\%), a high value as among the six highest futures (5\%), and a mid value as among middle 24 futures (40-60\%). There exist more than 3.8 billion unique combinations of six futures from the ensemble of 121 futures. Using a relatively crude but easy to implement search procedure, the analysts randomly tested combinations of futures for approximately 180 computing hours. The search found 31 alternative six-future sets that met the criteria of at least one low, mid, and high CMI value for each basin.

To choose among the 31 candidate sets, the report ranked the sets by the maximum number of basins with either a high or low CMI value in any single climate future. This criterion enabled the analysts to explore the implications of climate correlations among the basins. They identified one preferred set, which included a climate sequence that had "high" CMI in five basins.

\subsection{Combining Vulnerability and Diversity}

The ECRAI report combined diversity and vulnerability criteria in selecting its set of six representative climate futures from the original ensemble of 121 futures. The categorization of low, mid, and high CMI values represented the policy-relevant vulnerabilities in that low CMI presents a risk of over-investment in hydropower and irrigation infrastructure while high CMI represents a risk of under-investment. The requirement that any acceptable set of six futures 


\section{SECOND REVISED VERSION}

sample at least one low, mid, and high CMI value for each basin, and the ranking of the acceptable sets by the maximum number of high or low CMI values in any single future, represents a diversity measure. In this section we demonstrate a significantly more sophisticated combination of a vulnerability and diversity-based approach for selecting a small number of futures for a simplified version of the objectives from the ECRAI report. Our experiments were performed on a smaller ensemble of climate futures than the ECRAI data. In particular, this study considers three African river basins instead of seven and uses only climate projections from the CMIP3 ensemble (56 futures) rather than both the CMIP3 and CMIP5 ensembles (121 futures).

The experimental setup and terminology we use here are as follows. Using the term in the Morphological Analysis sense of the word, we regard a scenario as consisting of a model run with particular values for two input variables (Climate Model, Emissions) and the corresponding values for three output variables (the climate conditions in each of the river basins: Volta, Orange and Zambezi). Our experimental setup contained 22 different global climate models (identified as M1 - M22), three SRES (Nakicenovic et al. 2000) emissions scenarios (B1, A1B and A2).

Finallly, the three output variables have states corresponding to the three alternative climate conditions in each basin (Dry, Historical, or Wet). The states Wet, Hist, and Dry correspond to the CMI values of Low, Mid and High used in the ECRAI report (Cervigni et al. 2015). The complete list of climate models used is available from the authors upon request.

In the following, a scenario is formally defined as $S=\left(s_{\text {Model }}, s_{\text {Emission }}, s_{\text {Volta }}, s_{\text {Orange }}, s_{\text {Zambezi }}\right)$, with small $s$ being a state of the corresponding variable. Finally, a scenario set of $N$ scenarios is denoted by $S=\left\{S^{1}, S^{2}, \ldots, S^{N}\right\}$.Table 1 below illustrates the five variables and their associated states and the example scenario as a morphological field.

Table 1: Morphological field describing the five variables and their associated states. Note that the table is not a matrix, but represents the space of possible states for the variables in the morphological field (see e.g. Ritchey 2006). The table should be interpreted as follows: the variable 'Climate Model' can be one of 22 different states (M1 ... M22), the variable 'Emission' can be one of three states (A2, A1B, B1), the variable 'Volta' can be one of three states (Wet, Hist, Dry), etc. The possible scenario $S^{l}=(\mathrm{M} 1, \mathrm{~A} 1 \mathrm{~B}$, Wet, Hist, Hist) is illustrated in the table as gray cells. 


\begin{tabular}{|c|c|c|c|c|}
\hline Climate Model & Emission & Volta & Orange & Zambezi \\
\hline M1 & $A 2$ & Wet & Wet & Wet \\
\hline M2 & $A 1 B$ & Hist & Hist & Hist \\
\hline ... & B1 & Dry & Dry & Dry \\
\hline ... & & & & \\
\hline M22 & & & & \\
\hline
\end{tabular}

Our task is now to choose a scenario set consisting of six scenarios out of the 56 alternative model runs. This can be done in approximately 1.1 million different ways. First we reduce this very large number of combinations by adopting the criterion that the sets should contain at least one wet, one dry and one historical, for each basin. Choosing a set that contains all possible outcomes for each variable is a standard approach in scenario planning, and by using this criterion here we reduce the number of possible sets to approximately 290 000. In the sections below we will subsequently introduce more criteria and combinations of criteria.

The structure of this section is as follows. Section 3.2.1 introduces a measure related to vulnerability and scenarios that are 'optimal' with regards to this criterion are identified and the diversity of these sets is assessed. In section 3.2.2 a diversity measure is used for identifying optimal sets of scenarios without taking into account the previous criterion related to vulnerability. In section 3.3.3 an optimization problem taking into account the two previous criteria is defined. In the first three sub-sections 3.2.1-3 we only address the output variables (Volta, Orange, Zambezi). In Section 3.2.4 input variables (Climate Model, Emissions) are also incorporated into the analysis to make a full selection of scenarios.

\subsubsection{Maximally Extreme Sets of Climate Conditions}

The first additional criterion for defining optimal sets asks how extreme are the climate conditions in each scenario. This 'extremeness' can be seen as a policy-related measure, because 


\section{SECOND REVISED VERSION}

proposed investment policies may perform poorly in extremely wet or extremely dry futures. A set of scenarios with more extremes should be ranked higher than a set with fewer extremes. Here we use the following extremeness measure:

$$
E\left(S^{i}\right)=\left|s_{\text {Volta }}^{i}+s_{\text {Orange }}^{i}+s_{\text {Zambezi }}^{i}\right|
$$

where the states $\left(s^{i}\right.$ Volta etc.) of the river basins are defined as Dry $=-1$, Historical $=0$ and Wet $=$ 1. The intuition of this measure is that a scenario where all three basins are dry will get a high score in the search for a good future set, as will a scenario where all three basins are wet. This measure of extremeness was policy-relevant for the ECRAI report because it corresponds to situations in which risk sharing investments among river basins, such as inter-basin power transmission lines, are more or less useful. The extremeness measure used here was deemed relevant for the particular application, but other extremeness measures are possible.

Remember that our goal is to select a set of six different scenarios. We therefore define the mean extremeness of such a set of six scenarios as $E_{\text {mean }}(\boldsymbol{S})=\sum_{i=1}^{6} E\left(S^{i}\right) / 6$ and search for sets with maximum value of $E_{\text {mean }}$ in the 292514 possible sets in our experimental case. The result of this optimization on our experimental case is 10 sets, which all have the same maximum value of $E_{\text {mean }}$.

Given the discussion in the Introduction, and more specifically in Section 2.2, it is relevant to ask, how diverse are the scenarios in these 10 sets? We define a diversity measure (based on Equation (1) with $w_{k}=1$ reflecting the fact that diversity for all three basins are equally important) for the three output variables, as follows:

$$
D_{\text {output }}\left(S^{i}, S^{j}\right)=\left|s_{\text {Volta }}^{\mathrm{i}}-s_{\text {Voltal }}^{\mathrm{j}}\right|+\left|s_{\text {Orange }}^{i}-s_{\text {Orange }}^{j}\right|+\left|s_{\text {Zambezi }}^{i}-s_{\text {Zambezi }}^{j}\right| .
$$

With these measures in hand distances between all pairs of scenarios in a set are calculated. For each set consisting of six scenarios this means 15 distances in total $(n *(n-1) / 2)$. These distances are defined for pairs of scenarios. In a set with more than two scenarios it is possible to define the 


\section{SECOND REVISED VERSION}

minimum (cf. Equation (2) above) of all distances and the mean value of all the distances (cf. Equation (3) above).

This analysis shows that out of the 10 sets with maximum 'extremeness', 7 sets contain at least one pair of scenarios with $D_{\min }=0$, i.e. at least two scenarios are equal in the three output variables (all three river basins are the same). These sets do not qualify as optimal sets because we do not want two sets with the same output in a set of six scenarios. The remaining 3 candidates all have $D_{\min }=1$, i.e. at least two scenarios differ in only one state. In sum, the sets with maximal value of extremeness do not perform well with regard to scenario diversity.

\subsubsection{Maximally Diverse Sets of Climate Conditions}

In the previous section we saw that the result of choosing sets that were maximally extreme did not yield a very diverse set. In this section we instead identify a maximally diverse set of futures. When striving for diversity it makes sense to strive for high value of both the diversity measures (minimum value and mean value); we do not want any pair of futures to be too close to each other and we also want them all to be widely separated. We therefore base the objective function that we aim to maximize, $m_{1}(\boldsymbol{S})$, on a combination of the minimum and mean values of the set of output distances for the set:

$$
m_{1}(\boldsymbol{S})=\alpha D_{\min (\text { output })}(\boldsymbol{S})+(1-\alpha) D_{\text {mean }(\text { output })}(\boldsymbol{S})
$$

The parameter $\alpha$ represents the relative weight on minimum and mean distance respectively in the optimization. With this objective function the scenario diversity methodology is now used for generation of sets that are maximally diverse. For $\alpha=0.5$ the optimization returns 8 sets with maximum values of $D_{\text {min(output) }}=2$ and $D_{\text {mean(output) }}=3.33$. In this particular case there exist an $\alpha$ for which the maximal value of both minimum and the mean value is attained. While these sets are maximally diverse their 'extremeness', i.e. $E_{\text {mean }}$, is only 61 per cent of the maximum value calculated in section 3.2.1. The sets with the maximal value of diversity do not perform well with regard to extremeness. Hence, there exists a tension between extremeness and diversity. 


\section{SECOND REVISED VERSION}

\subsubsection{Combining Extremeness and Diversity}

This section combines the criteria of extremeness and diversity. An objective function $m_{2}$ combing the mean value of extremeness (Equation (4)) with the objective function for diversity (Equation (6)) is therefore defined as follows:

$$
m_{2}(\boldsymbol{S})=\beta\left(D_{\min (\text { output })}(\boldsymbol{S})+D_{\text {mean }(\text { output })}(\boldsymbol{S})\right)+(1-\beta) \frac{1}{6} \sum_{i=1}^{6} E\left(S^{i}\right),
$$

In order to simplify the comparison between the two objective functions $m_{1}$ and $m_{2}$, the two different diversity measures in Equation (6) have been given equal weight. Different choices of $\beta$ lead to sets emphasizing either high diversity (high $\beta$ ) or high 'extremeness' (low $\beta$ ). Figure 2 shows the values of $E_{\text {mean }}$ and the mean value of $D_{\min (\text { output) }}$ and $D_{\text {mean(output) }}$ (denoted D_output) for all (circa 290 000) scenario sets (each dot in the figure represents, in general, many scenario sets). Ideally, we would like a set of scenarios that are both as diverse as possible and as extreme as possible. However, in most cases, as in our example, a compromise between 'extremeness' and diversity has to be made. This can be accomplished in many different ways, as shown in Figure 1. 


\section{SECOND REVISED VERSION}

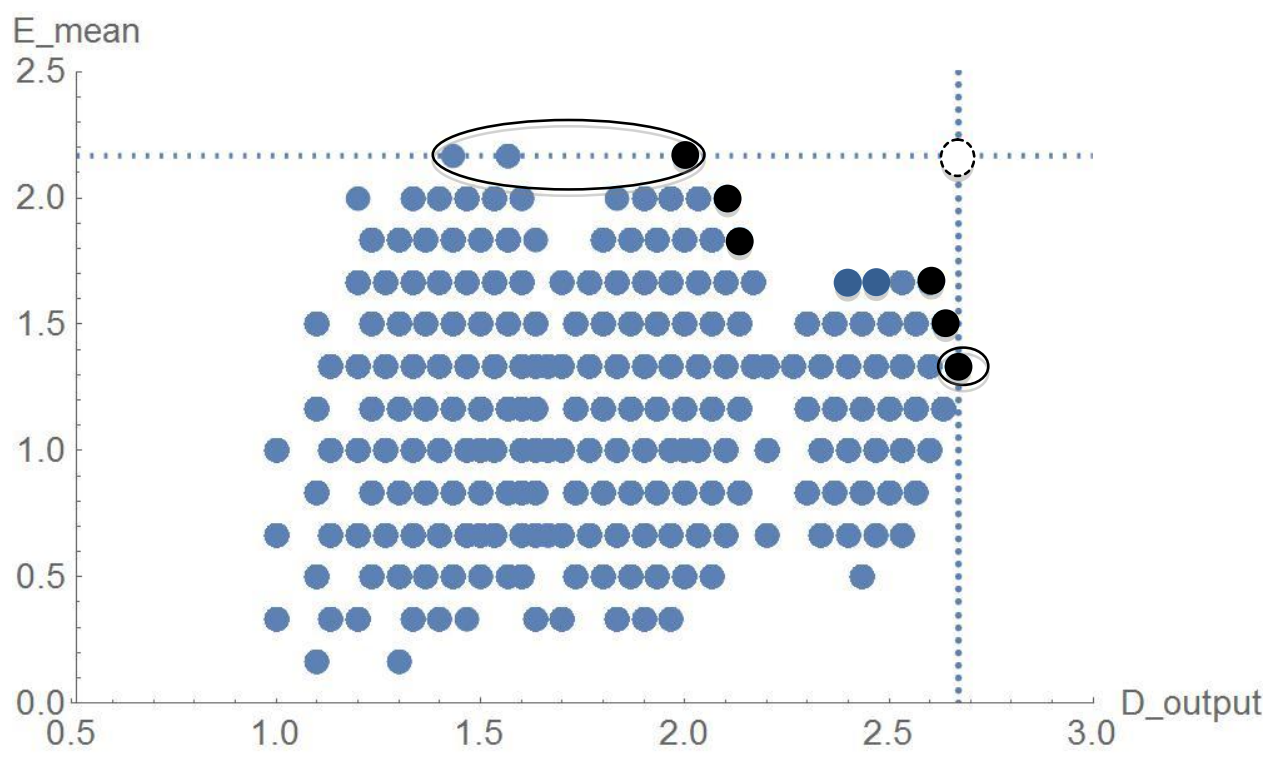

Figure 2: The values of extremeness $\left(E \_\right.$mean $)$and the mean value of the minimum diversity and mean diversity (D_output) for all possible sets in our experimental case. One dot in this figure represents one or more than one set with the same values. The upper three dots circled show the maximum extreme sets (with $\beta=0.25$ in Equation (7), see section 3.2.1) and the single rightmost dot circled shows the maximum diverse set (with $\beta=0.75$ in Equation (7), see section 3.2.2). The black dots show the pareto front in terms of extremeness and diversity (the pareto-optimal sets). The white dot with a dashed line shows the position of a hypothetical optimum with maximum extremeness and maximum diversity.

\subsubsection{Adding Diversity for Input Variables}

This last sub-section introduces diversity for the input variables (Climate Model, Emissions). Analogous to Equation (5), the distance between two scenarios $\left(S^{i}, S^{j}\right)$, measured by input variables, is given by:

$$
D_{\text {input }}\left(S^{i}, S^{j}\right)=\left|s_{\text {Model }}^{i}-s_{\text {Model }}^{j}\right|+\left|s_{\text {Emission }}^{i}-s_{\text {Emission }}^{j}\right|
$$

For the climate model variable we used a nominal scale for the distance between two different models. We set $\left|s_{\text {Model }}^{i}-s_{\text {Model }}^{j}\right|=0$ if the two scenarios use the same model and $\left|s_{\text {Model }}^{i}-s_{\text {Model }}^{j}\right|=$ 2 if scenarios use different models. The reason for setting it to 2 is to have the same distance as 


\section{SECOND REVISED VERSION}

for the other variables (see Section 2.2 and the discussion in relation to Eq. (1) above). For the emission variable we used an ordinal scale, where $B 1<A 2<A 1 B$. This ordering is motivated by the fact that until ca. 2060, the total radiative forcing of A1B is higher than that of A2 and the ECRAI analysis goes out to 2050 . We set $B 1=-1, A 2=0, A 1 B=1$. For the objective function to be maximized we chose a combination of measures for diversity of the input and output variables and of the measures for extremeness:

$$
\begin{gathered}
m_{3}(\boldsymbol{S})=(1-\gamma) \frac{1}{6} \sum_{i=1}^{6} E\left(S^{i}\right)+ \\
\gamma\left(\delta\left(D_{\min (\text { input })}(\boldsymbol{S})+D_{\operatorname{mean}(\text { input })}(\boldsymbol{S})\right)+(1-\delta)\left(D_{\min (\text { output })}(\boldsymbol{S})+D_{\operatorname{mean}(\text { output })}(\boldsymbol{S})\right)\right),
\end{gathered}
$$

The parameter $\gamma$ represents how much weight diversity should have compared to extremeness, and $\delta$ expresses the relative weight on input diversity vs. output diversity. Note that we have collected all diversity measures (inputs as well as outputs) within the $\gamma$ parameter. There are of course other ways of adding diversity for the input variables to Equation (7), for instance by grouping separately output variables (diversity and extremeness) and input variables (diversity only).

The optimum when maximizing Equation (9) for different values of the parameters $\gamma$ and $\delta$ is typically not unique and the number of possible sets of scenarios for each combination of parameters varies between 1 and approximately 30. The optimum is however sharper for this more complicated objective function compared to previous sections. Note that for some optimal scenario sets where $\gamma$ and $\delta$ are equal to 0 or 1 , the values of the diversity and extremeness measure need not be the same although the objective function $\left(m_{3}\right)$ takes the same value. 


\section{SECOND REVISED VERSION}

We implemented the equations above and made the optimization using the commercial software Mathematica. ${ }^{2}$ The code we used to make the experiments in Mathematica is available from the authors on request.

Table 2 shows the result for four different optimizations. The parameters ( $\gamma$ and $\delta$ ) have been selected to find one scenario set that maximizes input diversity (first row), one scenario set that maximizes output diversity (second row) and one set that maximizes extremeness (third row). These three scenario sets illustrate that optimizing for different aims could yield different scenario sets. The table also includes a more balanced set (fourth row) that performs relatively well for all the measures.

\footnotetext{
${ }^{2}$ We implemented the equations in Mathematica version 10.3 using the package Combinatorica. The optimization of the objective function given by Equation (9) for each value of the parameters $\gamma$ and $\delta$ took about 30 minutes on a normal laptop computer (Intel i7-3667U processor @ $2.00 \mathrm{GHz}$ ).
} 


\section{SECOND REVISED VERSION}

Table 2: The values of the diversity and extremeness measures for three optimal sets and one balanced set: One which maximizes diversity of the input variables, one which maximizes diversity of the output variables and one set which maximizes extremeness. Note that for the sets with maximized extremeness, there is an alternative set (not shown) with higher values of input diversity $(2 ; 3.13)$ and lower values on output diversity $(0 ; 3.13)$.

\begin{tabular}{|c|c|c|c|c|c|c|}
\hline Objective & Resulting scenario set & $D_{\min (\text { input })}$ & $D_{\text {mean(input) }}$ & $D_{\min (\text { output })}$ & $D_{\text {mean(out) }}$ & $\boldsymbol{E}_{\text {mean }}$ \\
\hline $\begin{array}{l}\text { Max input } \\
\text { diversity } \\
(\gamma=\delta=1)\end{array}$ & $\begin{array}{l}\text { (m9, B1, hist, hist, wet), } \\
\text { (m12, B1, dry, dry, dry), } \\
\text { (m19, B1, wet, wet, hist), } \\
\text { (m1, A1B, hist, wet, wet), } \\
\text { (m4, A1B, wet, wet, hist), } \\
\text { (m6, A1B, hist, dry, dry) }\end{array}$ & 2 & 3.2 & 0 & 3.067 & 2 \\
\hline $\begin{array}{l}\text { Max output } \\
\text { diversity } \\
(\gamma=1, \delta=0)\end{array}$ & $\begin{array}{l}\text { (m12, B1, dry, dry, dry), } \\
\text { (m14, B1, dry, wet, dry), } \\
\text { (m9, A2, wet, dry, dry), } \\
\text { (m16, A2, hist, dry, wet), } \\
\text { (m22, A2, wet, dry, dry), } \\
\text { (m1, A1B, hist, wet, wet) }\end{array}$ & 2 & 2.87 & 2 & 3.33 & 1.33 \\
\hline $\begin{array}{l}\text { Maximum } \\
\text { Extremeness } \\
(\gamma=0, \delta=0.5)\end{array}$ & $\begin{array}{l}\text { (m12, B1, dry, dry, dry), } \\
\text { (m19, B1, wet, wet, hist), } \\
\text { (m12, A2, dry, dry, hist), } \\
\text { (m13, A2, dry, hist, dry), } \\
\text { (m1, A1B, hist, wet, wet), } \\
\text { (m6, A1B, hist, dry, dry) }\end{array}$ & $1(2)$ & $\begin{array}{l}2.933 \\
(3.13)\end{array}$ & $\begin{array}{l}1 \\
(0)\end{array}$ & $\begin{array}{l}3 \\
(3.13)\end{array}$ & 2.167 \\
\hline $\begin{array}{l}\text { A balanced set } \\
(\gamma=0.75, \delta= \\
0.25)\end{array}$ & $\begin{array}{l}\text { (m12, B1, dry, dry, dry), } \\
\text { (m14, B1, dry, wet, dry), } \\
\text { (m16, B1, wet, dry, dry), } \\
\text { (m7, A2, dry, hist, hist), } \\
\text { (m1, A1B, hist, wet, wet), } \\
\text { (m4, A1B, wet, wet, hist) }\end{array}$ & 2 & 3.133 & 2 & 3.2 & 1.667 \\
\hline
\end{tabular}

\section{Concluding discussion}

This section first presents some challenges in the practical application of the proposed approach for combining vulnerability and diversity-based scenarios methods. We then discuss the potential for also incorporating the consistency approach, another important technique for scenario selection. 


\section{SECOND REVISED VERSION}

\subsection{Some challenges of practical application}

Real world applications would often present cases more complicated than the example used in this paper. This section describes some of the resulting challenges, and how they might be overcome.

First, finding an appropriate balance between diversity and vulnerability may prove challenging in many applications. Our experiments show that maximizing diversity in a scenario set does not necessarily yield a maximally policy-relevant set. For example Section 3.2 shows a diverse set with lower average extremeness. A scenario set may thus need to balance between diversity and extremeness. In this simple example we successfully found scenario sets relatively high in both diversity and extremeness, both for the output variables only (section 3.2.3) as well as for both input and output variables (section 3.2.4). But in general, it may not always be obvious how to choose weights for the different optimization criteria (e.g. extremeness vs. diversity, or between input and output diversity). This challenge is common in multi-criteria analysis (Keeney and Raiffa 1993). In this study, we conducted sensitivity-tests, combined with our own judgments, to find appropriate weightings. But in general, a computer-aided tool could also be designed to render this process more accessible and transparent, making the choice of scenario set accessible to stakeholder deliberation. The ultimate measure of the significance (or not) of alternative weights would of course be the extent to which the alternative scenario sets produce different policy results.

Second, the vulnerability and diversity approach can also prove computationally prohibitive in some cases, depending on the size of the original scenario set. Section 3's case study involved only five variables, of which four had three states and the fifth one (Climate Model) had 22 states. This represents a relatively simple problem compared to many in the scenario literature. The number of possible scenarios is a multiplicative function of the number of states for each variable - a large but usually computationally feasible number. However, the number of possible sets of $\mathrm{k}$ scenarios grows much more quickly with the number of possible cases $n$ ( $n$ choose $k$ ). Since scenario diversity is a set property, its computation can prove very demanding. Faster computers and more sophisticated optimization algorithms can partially address this challenge, but scenario 


\section{SECOND REVISED VERSION}

diversity calculations will often face computational limits. In addition, this paper's case study used particularly simple vulnerable scenarios. Applications with less simple vulnerable scenarios would have more complicated morphological fields (e.g. Table 1), with many combinations of inputs inconsistent with any particular vulnerable scenario. This in turn could also increase the demands on the diversity optimization. As mentioned in Section 3.2.3 we used Mathematica in this case study for convenience. This was not the optimal choice computationally, although Mathematica (version 10.3) now includes relatively fast algorithms for discrete problems. But larger problems would require use of state-of-the-art solvers for linear optimization. Previously, we developed for scenario diversity analysis a software tool utilizing the proprietary CPLEX optimizer (of IBM), which is capable of handling larger problems than Mathematica. We have not yet, however, integrated this software with the vulnerability-based approach for scenario selection.

Reducing the size of the original scenario set offers one of the most effective ways of reducing the complexity of the optimization problem. Vulnerability-based approaches may prove useful here. Note that such approaches identify regions of uncertainty space rather than specific model runs as their scenarios. If an analysis first uses a vulnerability approach to partition the full uncertainty space into distinct regions and then uses a diversity approach to choose a set of representative scenarios, one from each region, the overall combinations could be reduced by an order of magnitude or more. ${ }^{3}$ This illustrates the potential importance of vulnerability-analyses to complement diversity analyses.

Finally, future work combining vulnerability and diversity-based scenario selection could usefully focus on supporting interactive, participatory engagements with decision makers. Exercises aimed at selecting policy-relevant sets of scenarios generally occur in some specific context, often within an already defined policy process. Such decision contexts are characterized by a unique set of factors such as norms, social practice, and methodological traditions. There is

${ }^{3}$ This is because for large $n$ and small $k\left(\begin{array}{l}n \\ k\end{array}\right) \cdot\left(\begin{array}{c}n \\ k \\ 1\end{array}\right)^{-k} \approx \frac{k^{k}}{k !}$, where the first term represents choosing k scenarios from $\mathrm{n}$ possible cases and the second term represents all possible combinations of choosing one scenario from each of $\mathrm{n} / \mathrm{k}$ clusters of cases. For $k=4$ this is $\approx 11$ 


\section{SECOND REVISED VERSION}

of course a need to acknowledge all those aspects when designing the methodology for decision analysis and support; there is no 'one size fits all' approach to the problem addressed in this paper. One particular issue here is the degree of expert-driven vs. the degree of stakeholder participation in a particular set-up. It is important to stress that it is rarely the case that automatically generated scenario sets (as demonstrated in this paper) could be used directly. It would often prove necessary to employ the computer-generated results to support human creativity à la Intuitive Logics (Bradfield et al. 2005, van der Heijden 2005).

\subsection{Next Steps: Incorporation of Consistency Approaches}

This paper compares and combines the approaches of selecting a small number of scenarios according to maximal scenario diversity or focusing on scenarios that illuminate vulnerabilities. Yet another approach to selecting a small number of scenarios is to screen for scenarios with strong self-consistency (Weimer-Jehle 2006). Self-consistency refers to focusing on scenarios with qualitative elements depicting circumstances that reinforce each other, and this approach would be especially relevant for exploring the human dimensions of climate change. As noted by von Reibnitz (1988), self-consistent scenarios can represent long-term or persistent trends as opposed to scenarios that come into being only occasionally or momentarily. Recent studies that are examples using the self-consistency approach include waste management scenarios in Europe (Saner et al. 2011; Meylan and Spoerri 2014), social drivers of juvenile obesity (Weimer-Jehle et al. 2012), and socio-economic scenario narratives for IPCC assessments (Schweizer and Kriegler 2012; Schweizer and O’Neill 2014). Potentially, consistency approaches may also be compatible with diversity and vulnerability approaches for selecting small numbers of scenarios. The idea of combining the self-consistency and diversity approaches has earlier been proposed by KempBenedict (2012).

The report that was the basis for the case study in this paper (the ECRAI report) focused primarily on uncertainties in future climate and much (though certainly not all) of what is known about the spatial and temporal correlations among future climate trajectories is contained within the climate models. The ECRAI study did not, however, consider a wide range of socio-economic futures, in part because of a lack of suitable models to create self-consistent sets of scenarios for 


\section{SECOND REVISED VERSION}

exploring such trends. Such exploration is made additionally difficult by the frequent need to consider the time dependence of scenarios. In particular, considering the path dependence of adaptation decisions can help avoid maladaptation (IPCC 2012). Thus studies such as ECRAI might benefit from scenarios represented not only as contingencies at specific points in time (i.e. weather extremes), but also as full pathways in which various socio-economic factors co-evolve over time. Even though the scenarios featured in this paper's case study employ variables and states that do not lend themselves to self-consistency analysis, below we describe why and how self-consistency approaches could be integrated.

Consistency approaches are most appropriate for analysis when there is "a wished-for but nonexistent theory of how events affect one another in a multidisciplinary context" (Helmer $1981,1)$. Thus it is unnecessary to use consistency approaches if it is possible to build a detailed mathematical model (such as a climate model). However, such simulations are lacking, or not very inclusive or accurate, for many important socio-technical trends. The value of investigating such socio-economic trends in order to better understand vulnerability, impacts, and adaptation has been made clear by the latest RCP and SSP scenario framework for climate change research (Ebi et al. 2014).

The ECRAI report focused on the climatic effects of the SRES B1/A1B/A2 scenarios. However, it is important to note that between now and 2050, socio-economic conditions will also evolve. This means that focusing on climate scenarios alone to anticipate potential socio-economic impacts under climate change provides only half the story. Conceivably, maldevelopment could still leave many people vulnerable to the impacts of extreme weather even with smaller amounts of committed climate change (i.e. B1 climate scenarios). Examples of maldevelopment include concentrating population centers in locations subject to climate hazards (e.g. drought) or failing to establish weather prediction systems and emergency communication systems for densely populated areas currently lacking them. That opportunity costs would be expected with different development strategies has already been demonstrated in preliminary research with integrated assessment models applying the new RCP-SSP framework. For example in a feasibility test of socio-economic conditions and their associated emissions profiles, Riahi (2015) was unable to reproduce the low emission scenario RCP2.6 using socioeconomic futures of "Regional Rivalry" (SSP3) (O’Neill et al. 2015). 


\section{SECOND REVISED VERSION}

A potential concern with consistency approaches stems from their results being dependent upon judgments about interrelationships between scenario variables. Our current understanding of these - especially for socio-technical systems - may be incomplete or anachronistic.

Theoretically this could result in overconfidence (e.g. excluding combinations as inconsistent that turn out to happen); however, there are techniques to address this concern (Coyle et al. 1994, Schweizer and Kriegler 2012). Thus an important direction for future work building on the foundation laid by this study is to also integrate self-consistency approaches with diversity and vulnerability approaches. Such integration might provide a means to incorporate multiple socioeconomic futures into a study such as ECRAI. For instance, one might use self-consistency analysis to consider the socioeconomic conditions underlying the SRES B1/A1B/A2 scenarios, thereby making explicit some of the additional socioeconomic pressures on the future water basin, the corresponding demands on irrigation and energy production, and any constraints on the availability of capital. While the diversity and vulnerability approaches push the analysis to find more extreme cases, self-consistency analysis could explain why more complex climate-society scenarios would be plausible.

From an analytical perspective, the relationship between the diversity, vulnerability, and consistency scenario approaches could be hierarchical, where the self-consistency analysis would be applied only to socioeconomic variables of interest. For socioeconomic relationships that are difficult to quantify or simulate, the self-consistency analysis would produce socioeconomic cases that could be another case variable to add to the diversity-vulnerability analysis, where a selection among both socioeconomic and climate cases would be made. Alternatively, the selfconsistency analysis could be applied sequentially after the diversity-vulnerability analysis, as it has the feature of indeterminacy. Potentially, many sets that perform desirably according to diversity-vulnerability criteria could be further distinguished by how self-consistent their socioeconomic conditions are.

Given the uncertainties regarding the challenges ahead, it is important to strive for broad coverage of future possibilities when constructing scenario sets. This is especially important when considering extreme scenarios of climate change (Rogelj et al. 2009, Betts et al. 2011, UNEP 2012). Climate change near the upper bound of current projections does not just imply larger incremental changes, but also incorporate increasing variability and extremes (IPCC 2012) 


\section{SECOND REVISED VERSION}

as well as the possibility of triggering tipping points, i.e. forcing the climate system beyond a critical threshold when the system changes from one stable state to another (this transition can be reversible or irreversible; Lenton et al. 2008, Collins et al. 2013). Given this, it becomes important to be able to assess the vulnerability of current policies and to be able to identify those futures in which the policies perform poorly. From a policy planning point of view, the possibility of extreme scenarios of climate change calls for planning methodologies that both incorporate a diverse span of possible futures as well as illuminate vulnerabilities of current strategies and policies.

\section{Acknowledgements}

Excluded to keep manuscript anonymous.

\section{References}

Alcamo, J., 2001. Scenarios as tools for international environmental assessments, European Environmental Agency, Copenhagen.

Alcamo, J., 2008. Chapter Six The SAS Approach: Combining Qualitative and Quantitative Knowledge in Environmental Scenarios. Development in Integrated Environmental Assessment 2, $123-150$

Amara, R., 1981. The futures field: searching for definitions and boundaries. The Futurist, 15, $25-29$.

Betts R.A, Collins M., Hemming D.L., Jones, C.D., Lowe, J.A., and Sanderson, M,G.. 2011. When could global warming reach $4^{\circ} \mathrm{C}$ ? Philosophical transactions. Series A, Mathematical, physical, and engineering sciences 369, 67-84.

Boehlert, B., Strzepek, K., and Groves, D., 2015. Climate Change Projections in Africa. In: Cervigni, R. , Liden, R., Neumann, J. E., and Strzepek., K. M. (eds.) Enhancing the Climate Resilience of Africa's Infrastructure: the water and power sectors. Washington, DC: World 


\section{SECOND REVISED VERSION}

Bank.Bishop, P., Hines, A., Collins, T., 2007. The current state of scenario development: an overview of techniques. Foresight 9, 5-25.

Bradfield, R., Wright, G., Burt, G., Cairns, G., and van der Heijden, K., 2005. The origins and evolution of scenario techniques in long range business planning. Futures 37, 795-812.

Börjeson, L., Höjer, M., Dreborg, K.-H., Ekvall, T. and Finnveden, G., 2006. Scenario types and techniques: Towards a user's guide. Futures, 38, 723-39.

Bryant, B.P., and Lempert, R.J., 2010. Thinking inside the box: A participatory, computerassisted approach to scenario discovery. Technological Forecasting and Social Change 77, 3449.

Bureau of Reclamation, 2012. Colorado River Basin Water Supply and Demand Study: Study Report. United States Bureau of Reclamation: 89. Available at:

http://www.usbr.gov/lc/region/programs/crbstudy/finalreport/Study\%20Report/CRBS_Study_Re port_FINAL.pdf (Accessed March 14, 2016).

Carlsen, H, Dreborg, K.H., Wikman-Svahn, P., 2013. Tailor-made scenario planning for local adaptation to climate change Mitigation and Adaptation Strategies for Global Change 18, 12391255.

Carlsen, H.C., Eriksson, E.A., Dreborg, K.H., Johansson, B., and Bodin, Ö., 2016. Systematic exploration of scenario space. Foresight 18, 59-75.

Cervigni, R., Liden, R., Neumann, J.E., and Strzepek., K. M. (eds.), 2015. Enhancing the Climate Resilience of Africa's Infrastructure: the water and power sectors. Washington, DC: World Bank.

Collins, M., et. al, 2013. Long-term Climate Change: Projections, Commitments and Irreversibility. In: Stocker, T.F., et al. Climate Change 2013: The Physical Science Basis. Contribution of Working Group I to the Fifth Assessment Report of the Intergovernmental Panel on Climate Change. Cambridge, United Kingdom and New York, NY, USA: Cambridge University Press. 


\section{SECOND REVISED VERSION}

Coyle, R.G., Crawshay, R., and Sutton L. 1994. Futures assessment by field anomaly relaxation: A review and appraisal. Futures 26 (1): 35-43.

Ebi, K.L., Hallegatte, S., Kram, T., Arnell, N.W., Carter, T.R., Edmonds, J., Kriegler, E., et al., 2014. A New Scenario Framework for Climate Change Research: Background, Process, and Future Directions. Climatic Change 122 (3): 363-372.

Gausemeier, J., Fink, A., and Schlake, O., 1998. Scenario Management: An approach to develop future potentials. Technological Forecasting and Social Change 59, 111-130.

Gerst, M.D., Wang, P., and Borsuk, M.E., 2013. Discovering plausible energy and economic futures under global change using multidimensional scenario discovery. Environmental Modelling \& Software 44, 76-86.

Godet, M., 2000. The Art of Scenarios and Strategic Planning: Tools and Pitfalls. Technological Forecasting and Social Change 65, 3-22.

Gordon, T. J., and Hayward, H., 1968. Initial Experiments with the Cross Impact Matrix Method of Forecasting. Futures 1 (2), 100-116.

Groves, D.G., Fischbach, J. R., Bloom, E., Knopman, D., and Keefe, R., 2013. Adapting to a Changing Colorado River: Making Future Water Deliveries More Reliable Through Robust Management Strategies. Santa Monica: RAND Corporation.

Groves, D.G., and Lempert, R.J., 2007. A new analytic method for finding policy-relevant scenarios. Global Environmental Change 17, 73-85.

Helmer, O.,1981. Reassessment of Cross-Impact Analysis. Futures 13 (5), 389-400.

Intergovernmental Panel on Climate Change (IPCC), 2012. Managing the Risks of Extreme Events and Disasters to Advance Climate Change Adaptation. A Special Report of Working Groups I and II of the Intergovernmental Panel on Climate Change. New York: Cambridge University Press. 


\section{SECOND REVISED VERSION}

Kahn, H. and Wiener, A. J., 1967. The Year 2000: A Frameworkfor Speculation on the Next Thirty-Three Years. Macmillan.

Keeney, R.L. and Raiffa, H., 1993. Decisions with multiple objectives: preferences and value trade-offs. New York: Cambridge university press.

Kemp-Benedict, E., 2012. Telling better stories: Strengthening the story in story and simulations. Environmental Research Letters 4, 041004.

Kok, K., Patel, M., Rothman, D.S., Quaranta, G., 2006. Multi-scale narratives from an IA perspective: Part II. Participatory local scenario development. Futures 38, 285-311.

Kwakkel, J.H., Auping, W.L., and Pruyt, E., 2013. Dynamic scenario discovery under deep uncertainty: the future of copper. Technological Forecasting and Social Change 80, 789-800.

Lempert, R.J. and Collins, M., 2007. Managing the Risk of Uncertain Threshold Responses: Comparison of Robust, Optimum, and Precautionary Approaches. Risk Analysis 27(4), 10091026.

Lempert, R.J., Popper S.W., and Bankes, S.C., 2003. Shaping the Next One Hundred Years : New Methods for Quantitative, Long-term Policy Analysis. Santa Monica, CA: RAND Corporation.

Lempert, R., 2013. Scenarios that illuminate vulnerabilities and robust responses. Climatic Change 117, 627-646.

Lenton, T.M., Held, H., Kriegler, E., Hall, J.W., Lucht, W., Rahmstorf, S., and Schellnhuber, H.J., 2008. Tipping Elements in the Earth's Climate System. Proceedings of the National Academy of Sciences 105 (6), 1786-1793.

Meylan, G., and Spoerri, A., 2014. Eco-efficiency assessment of options for metal recovery from incineration residues: A conceptual framework. Waste Management 34 (1), 93-100.

Millennium Ecosystem Assessment (2005). Ecosystems and Human Well-Being. Island Press, Washington, DC. 


\section{SECOND REVISED VERSION}

Nakicenovic, N., Alcamo, J., Davis, G., de Vries, B., Fenham, J., Gaffin, S., Gregory, K., Grübler, A., Jung, T.Y., Kram, T., La Rovere, E.L., Michaelis, L., Mori, S., Morita, T., Pepper, W., Pitcher, H., Price, L., Riahi, K., Roehrl, A., Rogner, H.-H., Sankovski, A., Schlesinger, M., Shukla, P., Smith, S., Swart, R., van Rooijen, S., Victor, N., Dadi, Z., 2000. Special Report on Emissions Scenarios. New York: Cambridge University Press.

O’Neill, B.C., Kriegler, E., Ebi, K. L., Kemp-Benedict, E., Riahi, K., Rothman, D.S., van Ruijven, B.J., et al. 2015. The Roads Ahead: Narratives for Shared Socioeconomic Pathways Describing World Futures in the 21st Century. Global Environmental Change. in press.

Parker, A.M., Srinivasan, S., Lempert, R.J. and Berry, S., 2015. Evaluating Simulation-Derived Scenarios for Effective Decision Support. Technological Forecasting and Social Change 91, 6477.

Riahi, K., 2015. Overview of the SSP-Based Quantitative Projections and the Use of SPAs. presented at the IPCC Expert Meeting on Scenarios, Laxenburg, Austria, May 18.

Ritchey, T., 2002. General Morphological Analysis: A General Method for Non-Quantified Modeling. Available at: http://www.swemorph.com/pdf/gma.pdf (Accessed 14 Jun, 2015).

Ritchey, T., 2006. Problem Structuring Using Computer-Aided Morphological Analysis. Journal of the Operational Research Society 57 (7), 792-801.

Robinson, J.B., 1982. Energy backcasting A proposed method of policy analysis. Energy policy, 10(4), 337-344.

Rogelj, J., Hare, B., Nabel, J., Macey, K., Schaeffer, M., Markmann, K, and Meinshausen, M., 2009. Halfway to Copenhagen, no way to $2^{\circ} \mathrm{C}$. Nature Climate Change $3,81-83$.

Rounsevell, M.D.A., and Metzger, M.J., 2010. Developing qualitative scenario storylines for environmental change assessment. WIREs Climate Change 1, 606-619.

Saner, D., Blumer, Y.B., Lang, D.J., and Koehler, A. 2011. Scenarios for the implementation of EU waste legislation at national level and their consequences for emissions from municipal waste incineration. Resources, Conservation and Recycling 57, 67-77. 


\section{SECOND REVISED VERSION}

Scholz, R.W., Tietje, O., 2002. Embedded Case Study Methods: Integrating Quantitative and Qualitative knowledge. Thousand Oaks: Sage.

Schwartz, P., 1996. The Art of the Long View - Planning for the Future in an Uncertain World. New York, NY: Currency-Doubleday.

Schweizer, V.J., Kriegler, E., 2012. Improving environmental change research with systematic techniques for qualitative scenarios. Environmental Research Letters 7, 044011.

Schweizer, V.J., O’Neill, B.C., 2014. Systematic construction of global socioeconomic pathways using internally consistent element combinations. Climatic Change 122, 431-445.

Tietje, O., 2005. Identification of a small reliable and efficient set of consistent scenarios. European Journal of Operational Research 162, 418-432.

UNEP, 2012. Emissions Gap Report 2012: A UNEP Synthesis Report. Available via: http://www.unep.org/pdf/2012gapreport.pdf (Accessed 17 Jun 2015).

van der Heijden, K., 1996/2005. Scenarios: The Art of Strategic Conversation. 2nd. ed. Chichester, UK: John Wiley \& Sons.

van Vuuren, D.P., Edmonds, J., Kainuma, M., Riahi, K., Thomson, A., Hibbard, K., Hurtt, G.C., Kram, T., Kray, V., Lamarque, J.F., 2011. The representative concentration pathways: an overview. Climatic Change 109, 5-31.

Von Reibnitz, U., 1988. Scenario Techniques. Hamburg, New York: McGraw-Hill.

Weimer-Jehle, W., 2006. Cross-impact balances: a system-theoretical approach to cross-impact analysis. Technological Forecasting and Social Change 73, 334-361.

Weimer-Jehle, W., Deuschle, J., and Rehaag, R., 2012. Familial and societal causes of juvenile obesity - a qualitative model on obesity development and prevention in socially disadvantaged children and adolescents. Journal of Public Health 20 (2), 111-124. 


\section{SECOND REVISED VERSION}

Wilkinson A, R. Kupers, D. Mangalagiu 2013. How plausibility-based scenario practices are grappling with complexity to appreciate and address 21 st century challenges. Technological Forecasting and Social Change 80, 699-710.

Zwicky, F., 1969. Discovery, Invention and Research Through the Morphological Approach. New York: Macmillian. 